\title{
Connect \& Drive: design and evaluation of cooperative adaptive cruise control for congestion reduction
}

\author{
Jeroen PLOEG ${ }^{1 *}$, Alex F.A. SERRARENS ${ }^{2}$, Geert J. HEIJENK ${ }^{3}$ \\ 1. TNO Automotive, P.O. Box 756, 5700 AT Helmond, The Netherlands \\ 2. DTI Automotive Mechatronics, Croy 46, 5653 LD Eindhoven, The Netherlands \\ 3. Faculty of Electrical Engineering, Mathematics and Computer Science, Twente University, 7500 AE Enschede, The Netherlands
}

\begin{abstract}
Road throughput can be increased by driving at small inter-vehicle time gaps. The amplification of velocity disturbances in upstream direction, however, poses limitations to the minimum feasible time gap. This effect is covered by the notion of string stability. String-stable behavior is thus considered an essential requirement for the design of automatic distance control systems, which are needed to allow for safe driving at time gaps well below $1 \mathrm{~s}$. Using wireless inter-vehicle communications to provide real-time information of the preceding vehicle, in addition to the information obtained by common Adaptive Cruise Control (ACC) sensors, appears to significantly decrease the feasible time gap, which is shown by practical experiments with a test fleet consisting of six passenger vehicles. The large-scale deployment of this system, known as Cooperative ACC (CACC), however, poses challenges with respect to the reliability of the wireless communication system. A solution for this scalability problem can be found in decreasing the transmission power and/or beaconing rate, or adapting the communications protocol. Although the main CACC objective is to increase road throughput, the first commercial application of CACC is foreseen to be in truck platooning, since short distance following is expected to yield significant fuel savings in this case.
\end{abstract}

Key words: cooperative adaptive cruise control (CACC); string stability; cooperative driving; traffic congestion; fuel efficiency

(C) 2011 JMT. All rights reserved.

\section{Introduction}

$\mathrm{T}$ he main societal and technical problems stemming from mobility are congestion, carbon dioxide emissions, and safety. Actually, conventional Adaptive Cruise Control (ACC) systems are the first generation of driver assistance systems having the potential to influence traffic flow characteristics, though with limited benefits [1]. This is not surprising, since ACC has primarily been developed as a comfort system. As a consequence, relatively large inter-vehicle distances are employed, with a standardized minimum of $1 \mathrm{~s}$ time gap [2], the latter defined as the inter-vehicle distance divided by the follower vehicle velocity.

Time headways significantly smaller than $1 \mathrm{~s}$ are expected to significantly increase traffic throughput while decreasing fuel consumption and emissions [3-4]. At these small time gaps, however, ACC is known to be string unstable [5], meaning that perturbations such as

Received Jul. 7, 2011; revision accepted Aug. 15, 2011

*Corresponding author. E-mail: jeroen.ploeg@tno.nl (J. PLOEG)

(C) 2011 JMT. All rights reserved doi: 10.3969/j.issn.2095-087X.2011.03.009 short braking actions introduced by a platooning vehicle, will be amplified in upstream direction. As a result, fuel consumption and emissions will increase, and so-called ghost traffic jams may occur, negatively influencing throughput, whereas safety might be compromised as well. In order to prevent string instability, the well known ACC system, commonly based on forward looking radar or scanning laser (lidar), can be extended with a wireless communication link with one or more preceding vehicles, so as to utilize the additional information exchanged via this link. The resulting functionality is called Cooperative Adaptive Cruise Control (CACC) or, sometimes, Semi-autonomous Adaptive Cruise Control.

A vast amount of literature regarding control system design for CACC is available, see, e.g., [5-6] and the literature references contained therein. The focus of most research, however, is often on theoretical analysis rather than on the practical implementation and the evaluation thereof. Therefore, the HTAS-funded Connect \& Drive project has been initiated in 2009 , aiming to investigate the feasibility of CACC in view of large scale practical implementation. Participating companies and universities in Connect \& Drive are Small Advanced Mobility, TNO, Fourtress, Centric Tsolve, Twente Institute of 
Wireless and Mobile Communications, Eindhoven University of Technology, University of Twente, and Delft University of Technology. The project covers a wide range of topics, including control design, wireless communications, in-vehicle computer platform and software design, and human-machine interfacing. Part of the developed concepts have been tested using a test fleet consisting of six passenger vehicles, depicted in Fig. 1.

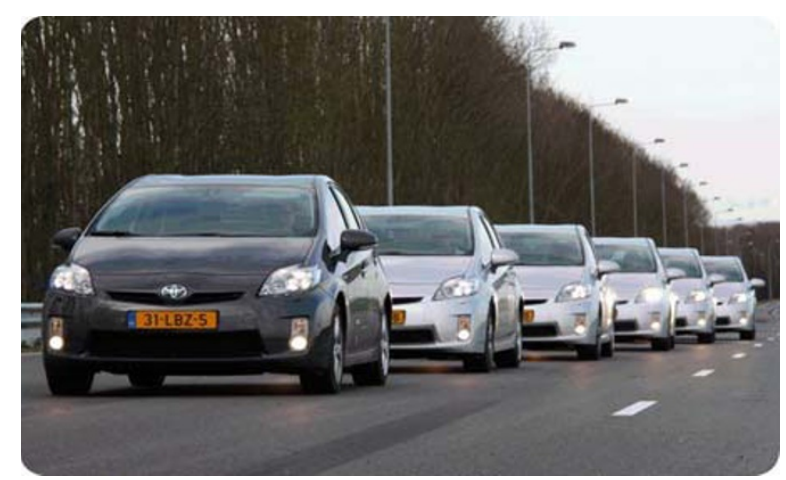

Fig. 1 The test fleet, consisting of six passenger vehicles
Since the Connect \& Drive project has been recently finalized, this paper presents some of the main results. To this end, the next section focuses on control system design for CACC. Next, Section 3 is dedicated to the enabling technology for CACC, being the inter-vehicle wireless communication link. Section 4 presents some experimental results, clearly indicating the advantages of CACC. Since large-scale deployment of such a system is not straightforward, carefully designed implementation roadmaps need to developed, as indicated in Section 0. Finally, Section 6 summarizes the main conclusions and proposes directions for further research.

\section{CACC system design}

The CACC control system designed in Connect \& Drive, consists of a number of functional components, as illustrated in Fig. 2.

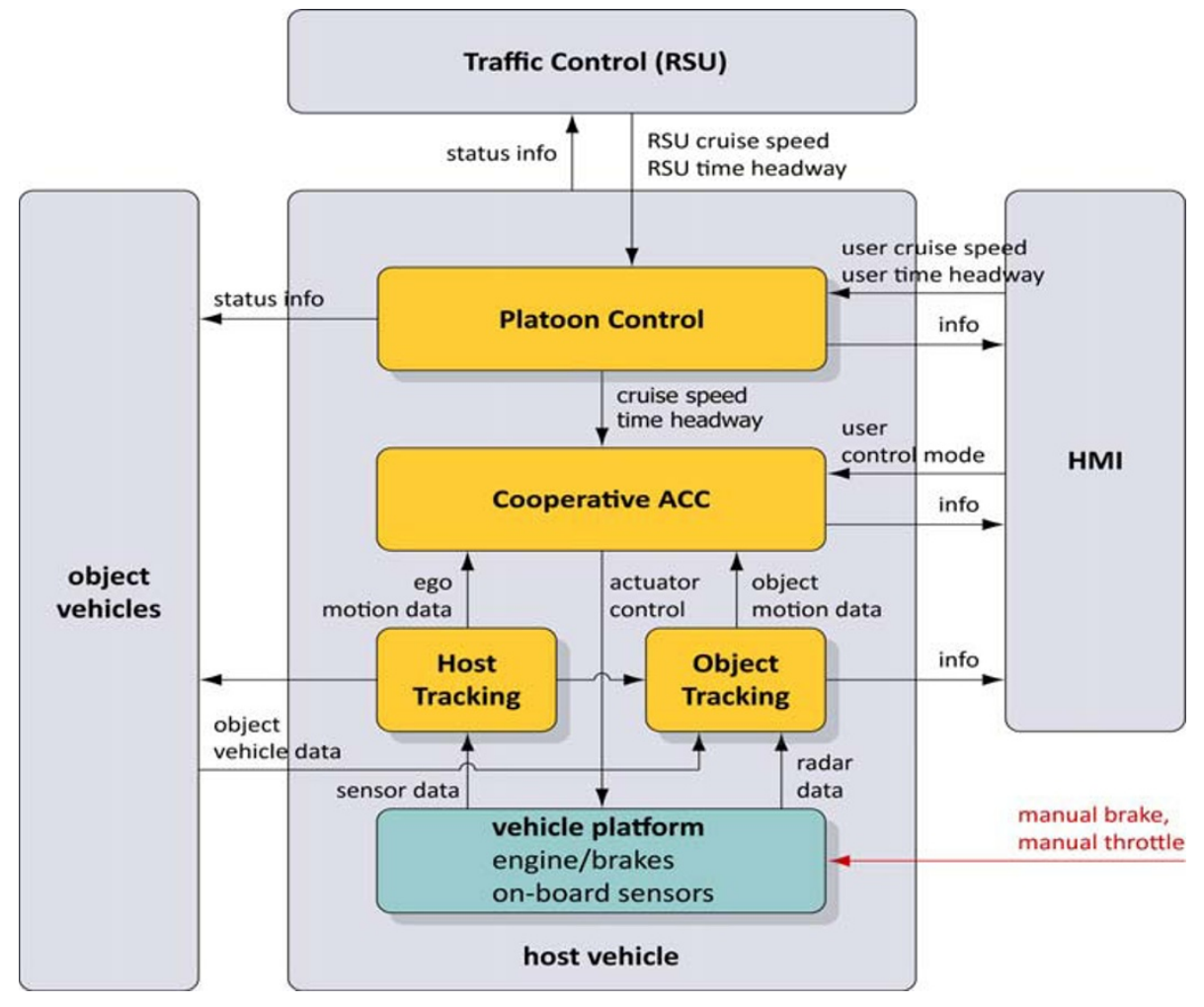

Fig. 2 Functional architecture of the CACC control system

Since the main control objective of CACC is to keep a desired distance (or time gap), it is necessary to be able to identify other object vehicles in the direct vicinity of the host vehicle and subsequently determine whether these vehicles are relevant, e.g., are driving on the same lane. To this end, two main sensors are available: the on-board radar, and a "virtual" sensor, i.e., the wireless link with other vehicles. The information that is transmitted over the wireless connection mainly consists of the vehicle motion state, being the position, velocity, and acceleration, as estimated by the so-called Host Tracking. Since the receiving vehicle is also equipped with a Host Tracking algorithm, it can calculate the relative motion in order to obtain the distance, the relative 
velocity and the relative acceleration of the object vehicle. This information is, to a certain extent, redundant with the information obtained by the on-board radar, which measures distance and relative velocity. The radar is still applied because of its accuracy, compared to the GPS-based position information that is communicated. Moreover, in case of packet loss (refer to Section 3), the radar is still able to provide the required information, except for the acceleration. The radar data and the ego motion data as estimated by the Host Tracking, are the input to the Object Tracking, which fuses both types of data in order to create a "world map", consisting of relevant vehicles and accompanying motion state. The motion state, in turn, serves as input to the actual CACC algorithm.

The CACC algorithm realizes a desired distance $d_{r, i}$ of vehicle $i$ to the preceding vehicle $i-1$ according to the constant time-headway spacing policy:

$$
d_{r, i}=r+h v_{i}
$$

where $h$ is the time headway, $v_{i}$ is the velocity of vehicle $I$, and $r$ is the standstill distance. This specific spacing policy is known to improve string stability [6]. The Object Tracking is the main information source for the CACC algorithm. One of the most important design choices concerns the vehicles taken into account in the control algorithm. Here, many possibilities exist, as illustrated in Fig. 3. This figure presents an overview of communication structures found in literature. In view of feasibility for practical implementation, the structure to be chosen preferably supports ad-hoc platooning, i.e., without an explicitly assigned platoon leader. Moreover, given possible scalability issues, a communication structure that only requires a limited range without the need for multi-hop strategies is desired. Hence, the onevehicle look-ahead structure is adopted, schematically depicted in the upper left side of Fig. 3.

Given the one-vehicle look-ahead strategy, the main reason to employ the wireless inter-vehicle communication link is to obtain information regarding the acceleration of the preceding vehicle since this cannot be measured by the radar. The preceding vehicle's acceleration is then used as a feedforward signal for the CACC controller. Consequently, without this feedforward, the CACC reverts to common ACC.

Mathematical details about the control design, including a string stability analysis, can be found in [7]. The string stability properties are illustrated in Fig. 4. This figure shows the response of a string of 10 vehicles to a velocity disturbance of the first vehicle without the use of wireless communication (ACC) as well as with CACC; in both cases a headway time $h=0.6 \mathrm{~s}$ is chosen. It is rather apparent that without wireless communication, the velocity perturbations increase in upstream direction which eventually will cause vehicles to closely approach each other, or even collide, without additional measures. This is a typical example of string-unstable behavior. In case of CACC, the follower vehicles not only react earlier, but also show a much better damped response, which is typical string-stable behavior. As a result, the traffic flow is stable and fuel consumption is decreased.
Preceding vehicle

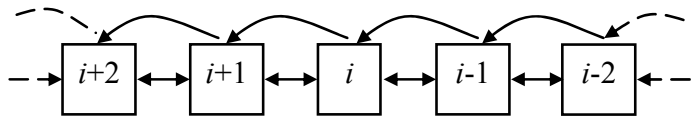

Leader vehicle

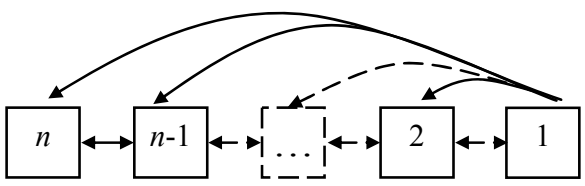

Preceding \& leader vehicle

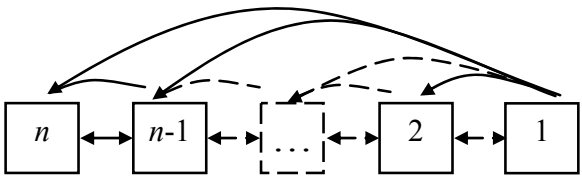

$n$ preceding vehicles

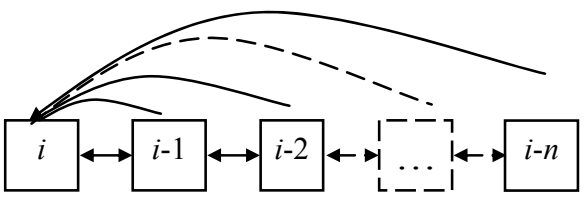

Mini platoons

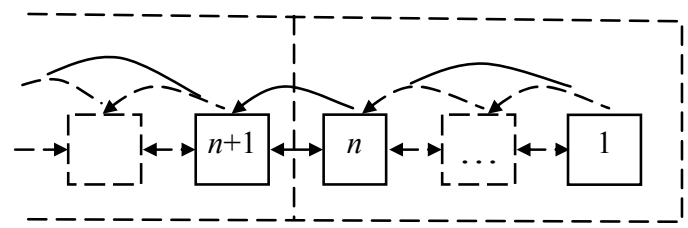

Preceding \& following vehicle (bidirectional)

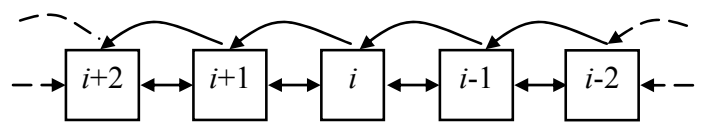

Driving direction

Fig. 3 Communication structures for CACC 


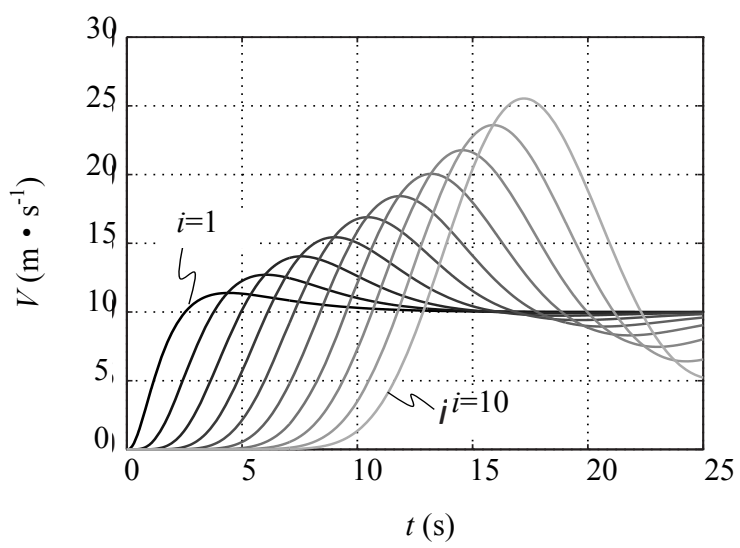

(a) Without wireless communication

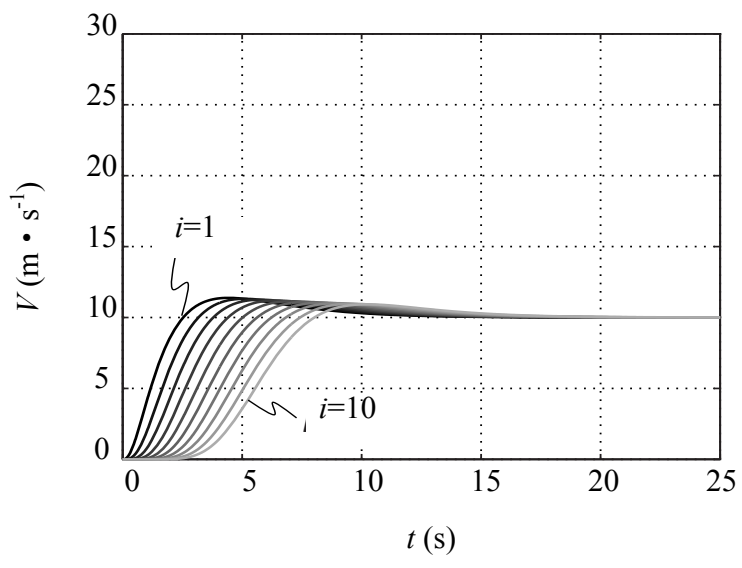

(b) With wireless communication

Fig. 4 Simulated velocity responses of a vehicle string

At the top level of the in-car CACC architecture (see Fig. 2), the so-called Platoon Control is located. This component is in fact the interface between the individual vehicle and the road side and is, therefore, capable of receiving a desired headway time and a desired cruise speed from a roadside unit (RSU). This functionality thus provides a means for external control so as to automatically influence the main CACC parameters, being velocity and headway time. In particular, the Platoon Control offers the possibility to automatically handle merging maneuvers at up-ramps [8] by creating sufficiently large gaps, initiated by the RSU.

Finally, it should be noted that the Human-Machine Interface plays an important role, since CACC effectively changes the role of the driver from actuator to supervisor. Hence, he should be adequately informed. The interested reader is referred to [9] and the references contained therein for further information.

\section{Ad-hoc wireless communications in a mobile environment}

In the Connect \& Drive project, wireless communications between vehicles and between RSUs and vehicles is based on the IEEE 802.11 standard. Versions of this standard that allow laptops, PDAs, etc., to wirelessly connect to access points are commonly known under the name WiFi. For vehicular environments, a special version of the standard, IEEE $802.11 \mathrm{p}$, has been defined for the $5.9 \mathrm{GHz}$ band to deal with the high speed of vehicles and the ad-hoc nature of the communications. Our design supports two different modes of communication, used for different purposes:

- Beaconing

In this mode of operation, all one-hop neighbors are periodically (10 times per second) informed about position, velocity, and acceleration of the transmitting vehicle, alongside some additional information. Additionally, RSUs can instruct passing vehicles regarding the advised time headway and the advised cruise speed.

- Constrained geocasting

In order to support merging maneuvers, messages about merging vehicles are forwarded over multiple hops to a set of vehicles that is expected to arrive at the merging area at approximately the same time as a merging vehicle. This set of vehicles is determined during the message forwarding operation, and depends, a.o., on the speed and location of vehicles. Constrained geocasting is further discussed in [8].

We have specified beaconing to support rates up to 25 beacons per second $(25 \mathrm{~Hz})$. A node is required to receive beacons from the closest 15 vehicles within a $200 \mathrm{~m}$ range ahead. Beacons should be received with a low latency (smaller than $200 \mathrm{~ms}$ ). Potentially, beaconing should operate in a congested multi-lane multihighway environment.

A potential problem for high-rate beaconing in vehicular networks is channel overload. Since beacons are transmitted as 802.11 broadcasts, they are not acknowledged. As a result, no retransmissions of lost beacons take place. Therefore, the transmitter does not receive any feedback with respect to success or failure of a transmission, so that the standard reaction to overload (increase of the so-called contention window) does not take place. Furthermore, in a vehicular environment, there may be many hidden nodes, i.e., nodes that do not sense an ongoing transmission, and start transmitting to the same receiver, resulting in packet loss. An exploration of this problem is performed in [10], showing that as a result of the mentioned factors, many beacons may not be received by their intended receivers. Consequently, the beacon reception rate is much lower than the beacon transmission rate as illustrated in Fig. 5 .

This figure depicts the reception rate as a function of the transmission rate and the number of nodes, clearly showing that the reception rate might not benefit from an increased transmission rate in case of more than about 50 nodes. Solutions to this problem can be found 


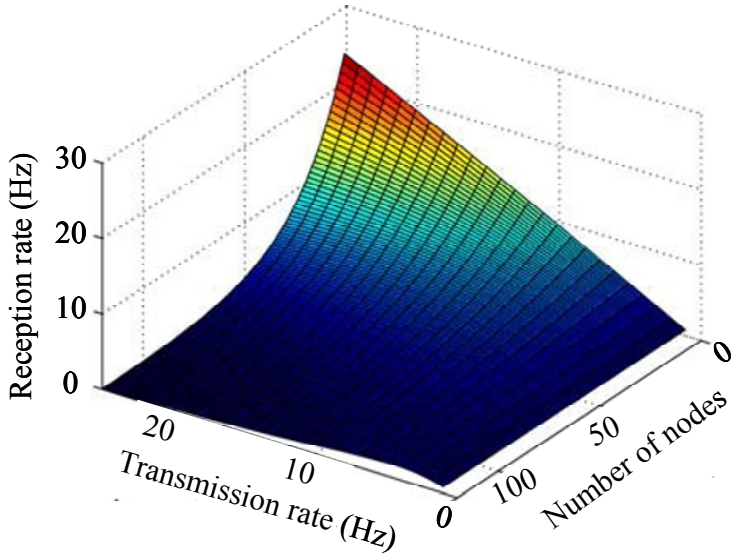

Fig. 5 Beacon reception rate from one target vehicle as a function of the beacon transmission rate and the number of vehicles within communication range. See [10] for more details.

in dropping beacons that have not yet been successfully transmitted when a newer beacon is ready for transmission, configuring a larger contention window [11], or adapting the transmission power and/or beaconing rate, depending on the state of the channel and the application needs, see, e.g., [12-13].

In the experiments described in this paper, the scalability problem addressed above does not appear due to the limited number of vehicles used in the experiments. We have adopted a $10 \mathrm{~Hz}$ beaconing rate, with some random offset to avoid repetitive collisions between beacons. For practical reasons, we have used the $2.4 \mathrm{GHz}$ band with IEEE $802.11 \mathrm{~g}$ for communications. The formatting of the payload of the beaconing messages has been adapted from Cooperative Awareness Message (CAM) format, specified by the COMeSafety project [14]. In the described experiments, only beacons received from the preceding vehicle are processed.

\section{Practical experiments}

To validate the designed controller, experiments are performed using the test fleet. To this end, CACC as well as ACC (i.e., CACC without the wireless link) are evaluated to compare the performance of both control systems. The test trajectory is defined by the desired acceleration $a_{\mathrm{ref}, 1}(t)$ of the lead vehicle, and consists of a constant acceleration of $0.5 \mathrm{~m} / \mathrm{s}^{2}$ form standstill to $70 \mathrm{~km} / \mathrm{h}(19.44 \mathrm{~m} / \mathrm{s})$, after which the desired acceleration is set to zero. In view of a high level of reproducibility, the lead vehicle is not manually driven, but instead has been equipped with a velocity controller. The desired velocity $v_{\text {ref, } 1}(t)$ is determined through integration of $a_{\mathrm{ref}, 1}(t)$, while using the latter as a feedforward signal. In [7], it is shown that for a time headway $h=0.7 \mathrm{~s}$, the CACC controlled system is just string stable, which is why this time headway has been adopted during the tests. Note that smaller time headways are possible, provided that the current latency of the wireless link (about $150 \mathrm{~ms}$ ) is decreased [7].

Fig. 6 shows the results of one of the tests. Fig. 6(a), showing the velocity response of the six vehicles in case of ACC, clearly illustrates the fact that this controller leads to string-unstable behavior: after the transition from constant acceleration to constant velocity, the overshoot of each consecutive vehicle grows. The amount of overshoot is still limited, but with longer strings, it would ultimately grow unbounded, theoretically. Fig. 6(b), showing the CACC response, is exemplary for string stable behavior, where the overshoot of follower vehicles is smaller than or equal to the lead vehicle overshoot.

Another remarkable aspect can be seen at startup of the vehicles. In case of ACC, it takes a considerably longer period of time before the last vehicle finally starts to accelerate, compared to CACC. From Fig. 6(a), it can be seen that the last vehicle starts to accelerate more than $14 \mathrm{~s}$ after the lead vehicle, whereas in Fig. 6(b), this

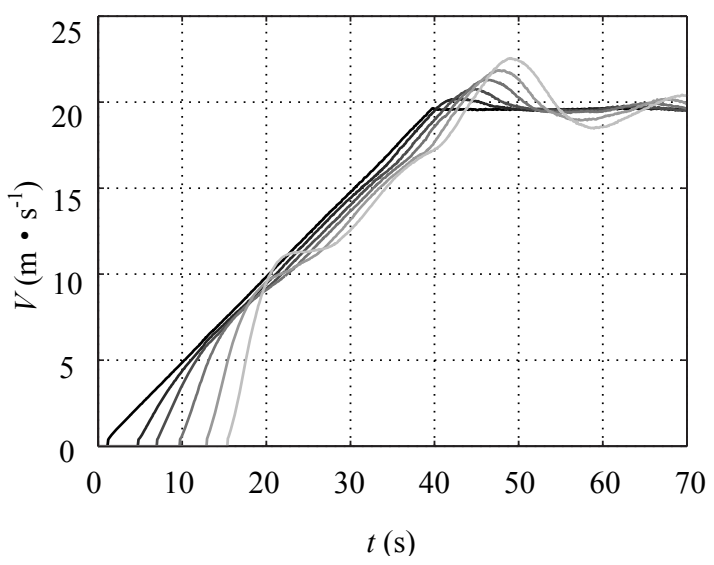

(a) Without wireless communication

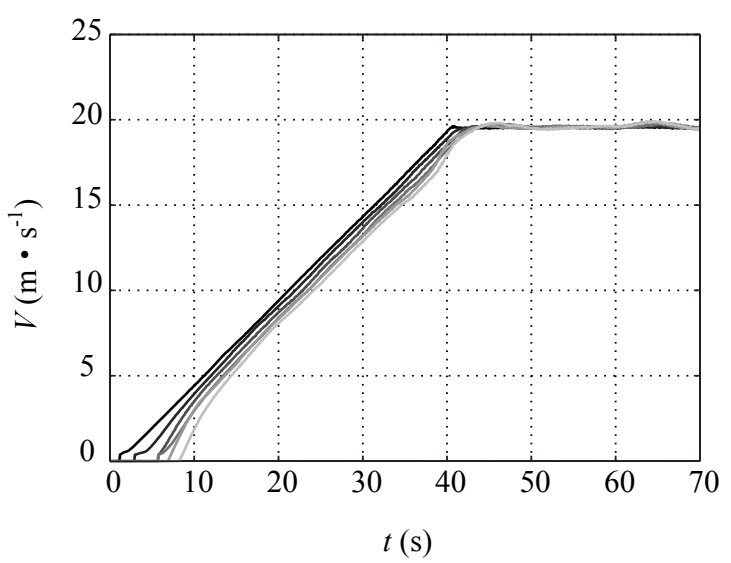

(b) With wireless communication

Fig. 6 Measured velocity response of the test fleet 
is only $7 \mathrm{~s}$. Consequently, CACC is beneficial in urban scenario's as well, since it would significantly increase throughput at traffic lights.

\section{Deployment roadmaps}

As described in the previous sections, CACC is an effective way to greatly increase vehicle string stability over ACC vehicles, whereas the latter establishes a disturbance damping being even lower than traditional pedal-controlled vehicles. The string stability increase in CACC mode enables a much shorter and more stable inter-vehicle following distance than currently seen in highway systems. Given such improved vehicle string performance, what practical applications could CACC (and further improved versions thereof) have in the near future? The rationale behind the Connect \& Drive project as well as the title of this paper refers to deployment of CACC for congestion reduction. For such congestion to really reduce, many cars must be equipped with CACC, e.g., with vehicle-to-vehicle (V2V) communication means. This will require years of penetration ramp-up, including the harmonized selection of communication protocol and technology standards within the automotive industry as a whole. The target communication protocol for V2V is IEEE 802.11p, whereas for vehicle-toinfrastructure (V2I) or vice-versa, this would be $3 \mathrm{G}$ (and beyond), see [15] and the references therein.

In order to realistically foresee the wide-spread deployment of CACC in the next 5 to 10 years, it is of utmost importance that a first (automotive) application market is strategically selected as well as a quick and successful deployment thereof. Furthermore, it is important that a solid roadmap is defined along which a sustaining growth in applications and penetration of CACC can emerge.

As a launching application market for CACC we have selected "road trains", e.g., platoons of long-haul freight trucks, as illustrated in Fig. 7, for cross-border transportation of goods, particularly in a European context. The motivations for this selection are the following.

- Long-haul trucks can collectively save a substantial amount of fuel (over $10 \%$ per truck) if they can drive (safely) at short inter-vehicle distances and

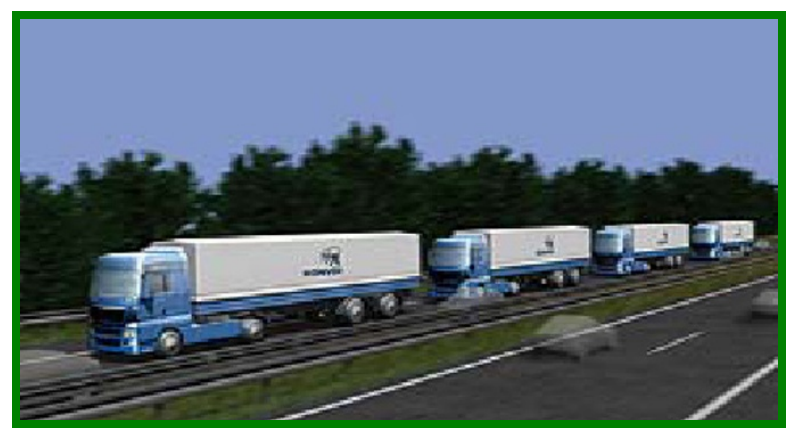

Fig. 7 First application of CACC: truck platooning in road trains thus benefit from lower air drag losses. Such high fuel savings are both beneficial for the environment as well as the business proposition of the fleet owner.

- Advanced driver support such as CACC is particularly interesting for professional drivers who drive many hours every day.

- Trucks have an electronically limited top speed by law, which naturally clusters them on the right highway lane. Next to that, truck drivers already have a professional approach to cooperative driving on the highway.

- Through V2V communications in combination with map-based information, long truck platoons can actually have coordinated behavior at exits and inroads in order to let other vehicles cross the truck lane, which can include (backward) rejoining the platoon afterwards. This is commonly referred to as "platoon tactics".

- AEBS (Advanced Emergency Braking System) has to be on every new European truck in 2015 by law. AEBS incorporates environment sensors (radar, camera) as well as a computerized link between these environment sensors and the drive/brake system of the truck. Given the infrastructure of this highly integrated emergency system, it establishes an excellent carrier for introduction of, and electronic fusion with, CACC.

- The truck market has advanced aftermarket and retrofit market service channels which could enable a swift introduction of fleet CACC, even though it might not have been deployed by the truck industry already.

- The truck industry is more homogenous in product specifications and performance parameters than the much more diverse passenger car industry. For a large part this is caused by pan-European regulations.

The aim is to introduce the first truck CACC systems shortly after the introduction of AEBS in 2015. The next target markets are electric and plug-in hybrid cars, as illustrated in Fig. 8, which benefit from the vehicle speed being as constant as possible to increase electric driving range (or reduce the size of the expensive battery pack). Next to that, they have a highly computerized and integrated driving/braking system for maximization of brake energy recovery and other hybrid functions. CACC can be relatively easy integrated with such platforms. Owners of such cars can be more easily triggered for further environmentally friendly solutions than an average car buyer. Along with the introduction of CACC for such cars, governments are triggered to establish separate green corridor lanes on the highways so that electric cars can benefit from both energy savings, relaxed driving as well as congestion free driving. Such attractive "modality" should convince the average car buyer to join, in effect greatly stimulating the future zero and ultra-low emission mobility through direct customer benefits. Finally, the large social networking trends on the internet can have repercussions in the mobility industry in this way. 


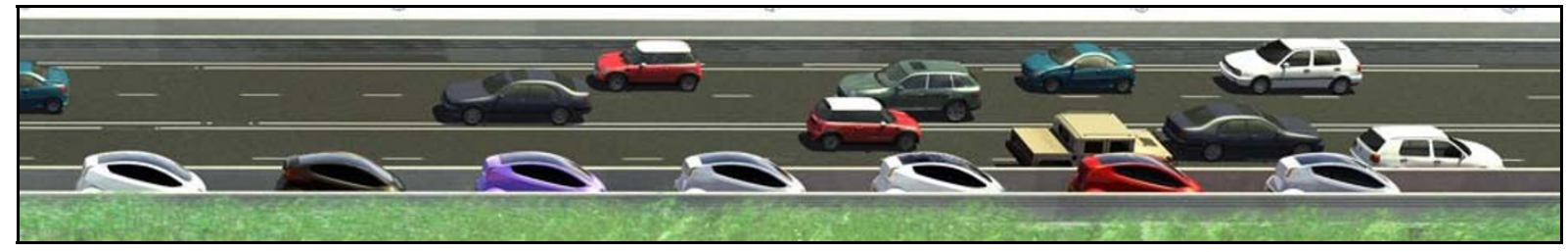

Fig. 8 Application outlook of CACC: automated and dense platoons of electric vehicles

\section{Conclusions}

String stability is an essential requirement for the design of vehicle following control systems that aim for short-distance following. It has been shown, by simulation and practical experiments, that $\mathrm{CACC}$, which is based on common ACC sensors and a wireless intervehicle communication link, allows for time gaps significantly smaller than $1 \mathrm{~s}$ while maintaining string stability. In the experimental set-up, consisting of a test fleet of six vehicles, a time headway of $0.7 \mathrm{~s}$ appeared to yield string-stable behavior whereas time gaps less than $0.5 \mathrm{~s}$ are feasible when optimizing the wireless link with respect to latency. As a result, a significant increase in road throughput and, in case of heavy-duty vehicles, decrease of fuel consumption and emissions can be expected.

Ongoing research is directed towards solving the scalability problem that arises at large-scale deployment of wireless communications. Several solutions are proposed here, such as adapting the transmission power and/or the beaconing rate. In parallel, much focus is put on creating business cases for CACC in order to accelerate commercial introduction. In this respect, application for truck platooning seems to provide a clear business case, since short inter-vehicle distances are expected to yield significant fuel savings for trucks.

\section{References}

[1] J. VanderWerf, S.E. Shladover, M.A. Miller, et al., Effects of adaptive cruise control systems on highway traffic flow capacity, Transportation Research Record, 2002, 1800(2): 7884.

[2] ISO 15622, Transport information and control systemsadaptive cruise control systems performance requirements and test procedures, International Organization for Standardization, Transport information and control systems, 2002.

[3] B. van Arem, C.J.G. van Driel, R. Visser, The impact of cooperative adaptive cruise control on traffic-flow characteristics, IEEE Transactions on Intelligent Transportation Systems, 2006, 7(4): 429-436.

[4] S.E. Shladover, Automated vehicles for highway operation (automated highway systems), In: Proceedings of the Institution of Mechanical Engineers, Part I: Journal of Systems and Control Engineering, 2005, 219(1): 5375.

[5] R. Rajamani, C. Zhu, Semi-autonomous adaptive cruise control systems, IEEE Transactions on Vehicular Technology, 2002, 51(5): 1186-1192.

[6] G.J.L. Naus, R.P.A. Vugts, J. Ploeg, et al., String-stable CACC design and experimental validation: a frequencydomain approach, IEEE Transactions on Vehicular Technology, 2010, 59(9): 4268-4279.

[7] J. Ploeg, B.T.M. Scheepers, E. van Nunen, et al., Design and experimental evaluation of cooperative adaptive cruise control, In: Submitted to the 14th International IEEE Conference on Intelligent Transportation Systems (ITS 2011), Washington D.C., 2011: 5-7.

[8] W.K. Wolterink, G.J. Heijenk, G. Karagiannis, Constrained geocast to support cooperative adaptive cruise control (CACC) merging, In: Proceedings of the Second IEEE Vehicular Networking Conference (VNC 2010), Jersey City, New Jersey, 2010: 41-48.

[9] R. Happee, M. Saffarian, J. Terken, et al., Human factors in the Connect \& Drive project, In: Proceedings of the 8th International Automotive Congress, Eindhoven, The Netherlands, 2011: 16-17.

[10] M. Van Eenennaam, W.K. Wolterink, G. Karagiannis, et al., Exploring the solution space of beaconing in VANETs, In: Proceedings of the First IEEE Vehicular Networking Conference, VNC2009, Tokyo, Japan, 2009: 28-30.

[11] R. Reinders, E.M. van Eenennaam, G. Karagiannis, et al., Contention window analysis for beaconing in VANETs, In: Proceedings of the Seventh IEEE International Wireless Communications and Mobile Computing conference, IWCMC 2011, Istanbul, Turkey, 2011: 5-8.

[12] M. Torrent-Moreno, J. Mittag, P. Santi, et al., Vehicleto-vehicle communication: fair transmit power control for safety-critical information, IEEE Transactions on Vehicular Technology, 2009, 58(7): 3684-3703.

[13] E.M. van Eenennaam, G. Karagiannis, G.J. Heijenk, Towards scalable beaconing in VANETs, In: Proceedings of the Fourth ERCIM Workshop on Emobility, Lulea, Sweden, 2010: 31.

[14] T. Kosch, I. Kulp, M. Bechler, et al., Communication architecture for cooperative systems in Europe, IEEE Communications Magazine, 2009, 47(5): 116-125.

[15] I. Jawhar, N. Mohamed, L. Zhang, Inter-vehicular communication systems, protocols, and middleware, In: Proceedings of the 5th IEEE International Conference on Networking, Architecture, and Storage, Macau, 2010: 282-287. 